\title{
Optical and digital processing I
}

\author{
Andrew G. Tescher \\ The Aerospace Corporation \\ P. O. Box 92957 \\ Los Angeles, California 90009

\section{David Casasent} \\ Department of Electrical Engineering \\ Carnegie-Mellon University \\ Pittsburgh, Pennsylvania 15213
}

Over one year ago, the concept of this special issue was first formulated. It was conceived, initially, as one special issue on digital image processing and a second special issue on optical data processing. These topics have been retained. However, rather than two separate issues, we have chosen to intersperse digital and optical papers throughout two issues. This has been done both for content and to emphasize the similarities in techniques and application areas between these two powerful computing disciplines. We trust that these truly joint-dual special issues will typify the rapidly increasing marriage of the disciplines of optical data processing and digital image processing.

The classic view that many have of optical processors is a matched-spatial filter frequency-plane-correlator. As the first paper by Caulfield, Aerodyne Research, et al. shows, optical pattern recognition has rapidly matured and grown in flexibility beyond the matched filter. Many of the new optical pattern recognition techniques described in this paper utilize extensive hybrid optical/digital technology and concepts whose origins lie in the digital image processing literature.

The first major contribution of optics was in synthetic aperture radar processing. In the paper by Ausherman from ERIM, the present status of digital and optical research in this area is summarized and the relative benefits of each are compared.

Digital image processing at Cal. Tech's Jet Propulsion Laboratory has always represented the latest in this technology. The paper by Green et al. presents the state of current technology in multiple image processing for planetary applications.

The paper by Mantock et al., of the University of Southern California's Image Processing Institute, describes a new hybrid pattern recognition technique for texture analysis using psuedocolor. This system is typical of how the disciplines of optical and digital processing can effectively complement one another.

The information content of imagery is important in both optical and digital systems. In the paper by Hall et al., University of Tennessee, abstract decomposition techniques are discussed whereby the ultimate information content of imagery may best be demonstrated and scene content analysis described.

Signal processing is an application area with real-time and parallel processing requirements most conducive for advanced data processing techniques. Psaltis and Casasent, CarnegieMellon University, describe a general formulation of optical signal processors in which the plethora of new architectures and algorithms can be classified and compared.

An obvious and well-advanced application areá for optical techniques is in data recording and storage. A summary of U.S., European, and Japanese advancements in this area is presented by Reddersen and Ralston from Harris Government Systems Group.

Finally, Neff summarizes the extensive program of the Air Force Office of Scientific Research in optical computing.

In the May/June 1980 Optical Engineering, several of these programs will be discussed in more depth, and other areas of common optical and digital research (e.g., transaxial tomography) will be described.

For further reading, the recent SPIE Proceedings on Applications of Digital Image Processing III (Volume 207), and Optical Pattern Recognition (Volume 201) are recommended. 